Rer. Saúde pübl., S. Paulo.

15:338-49. 1981

\title{
HIPOVITAMINOSE A EM COMUNIDADES DO ESTADO DE SÃO PAULO, BRASIL *
}

Maria José Runcada **

Donald Wilson **

Rosa Nilda Mazzilli **

Yaro Ribeiro Gandra**

RONCADA, M. J. et al. Hidovitaminose A em comunidades do Estado de São Paulo. Brasil. Rev. Saúde públ., S. Palllo. 15:338-49, 1981,

RESUMO: Foram realizados inquéritos nutricionais completos (de consumo de alimentos, bioquímico e clínico) referentes à deficiência de vitamina A em onze comunidades do Eslado de São Paulo. O inquérito alimentar mostrou consumo muito baixo de alimentos fontes de vitamina $A$, tanto de origem animal como vegetal. O inquérito bioquímico mostrou alta prevalência de níveis plasmáticos de vitamina A classificados como "deficiente" e "baixo". O inquérito clínico mostrou baixos coeficientes de prevalência para lesões oculares, principalmente as mais graves. Embora nem a cegueira, nem as lesōes oculares graves constituissem um problema de Saúde Pública. a maioria dessas populações corria o risco de que tais lesões viessem a se tornar um problema.

UNITERMOS: Vitamina A, deficiência. Inquéritos nutricionais. Estado de São Paulo, Brasil. Xeroftalmia.

\section{INTRODLCYAO}

Nunca é demais enfatizar a importância de estudos epidemiológicos regionais sobre deficiências nutricionais, especialmente a deficiência de vitamina $A-u m$ dos três tipos mais comuns de desnutrição.

No Brasil, muito poucos estudos desta natureza foram publicados em relação à hipovitaminose $A$, baseados em pesquisas populacionais incluindo consumo alimentar, e em exames clínicos e bioquimicos. Um dos mais conhecidos talvez seja aquele realizado pelo Interdepartmental Committee on Nutrition for National Development
(ICNND), em 1963', envolvendo seis dus nove estados do Nordeste brasileiro.

Com o objetivo de contribuir para 0 conhecimento do estado nutricional de outras regiões do Brasil, o Departamento de $\mathrm{Nu}$ triçāo da Faculdade de Saúde Pública da Universidade de São Paulo realizou, de 1969 a 1973, inquéritos nutricionais completos em várias comunidades do Estado de São Paulo, Brasil.

Os inquéritos foram realizados nas seguintes comunidades: Iguape, Icapara Pontal do Ribeira (Fig. $a, b, c$ ), em

* Trabalho realizado através de convénio com "Serviço do Vale do Ribeira (Departamento de Aguas e Energia Elétrica do Governo do Estado de São Paulo) e Secretaria dos Negócios da Saúde de Săo Paulo. Apresentado no XI Congresso Internacional de Nutriçăo. Rio de Janelro. Brasil, 1978 .

** Do Departamento de Nutrição da Faculdade de Saúde Pública da Universidade de São paulo - Ar Dr. Arnaldo. 715 - 01255 - Săo Paulo. SP - Brasil. 
RONCADA, M.J. et al. Hipovitaminose A em comlinidades do Estado de Sazo Paulo, Brasil. Rev. Saúde puibl., S. Paulo, 15:338-49, 1981.

1968; Apiai, Ribeira e Barra do Chapéu (Fig. $d, e, f$ ), em 1970; Embu-Guaçu e Getulina (Fig. $g$, h), em 1972; e, finalmente, Cipó, Morro Agudo e Salles Oliveira (Fig. $i, j, k$ ), em 1973.

As comunidades indicadas como $a, b, c$, $d, e$ e $f$ estão localizadas no Vale do Ribeira, as três primeiras na região litorânea. Essa região é uma das menos desenvolvidas do Estado de São Paulo.

Neste trabalho, analisaremos os resultados encontrados referentes aos inquéritos alimentar, bioquímico e clínico, no que diz respeito à vitamina $\mathrm{A}$.

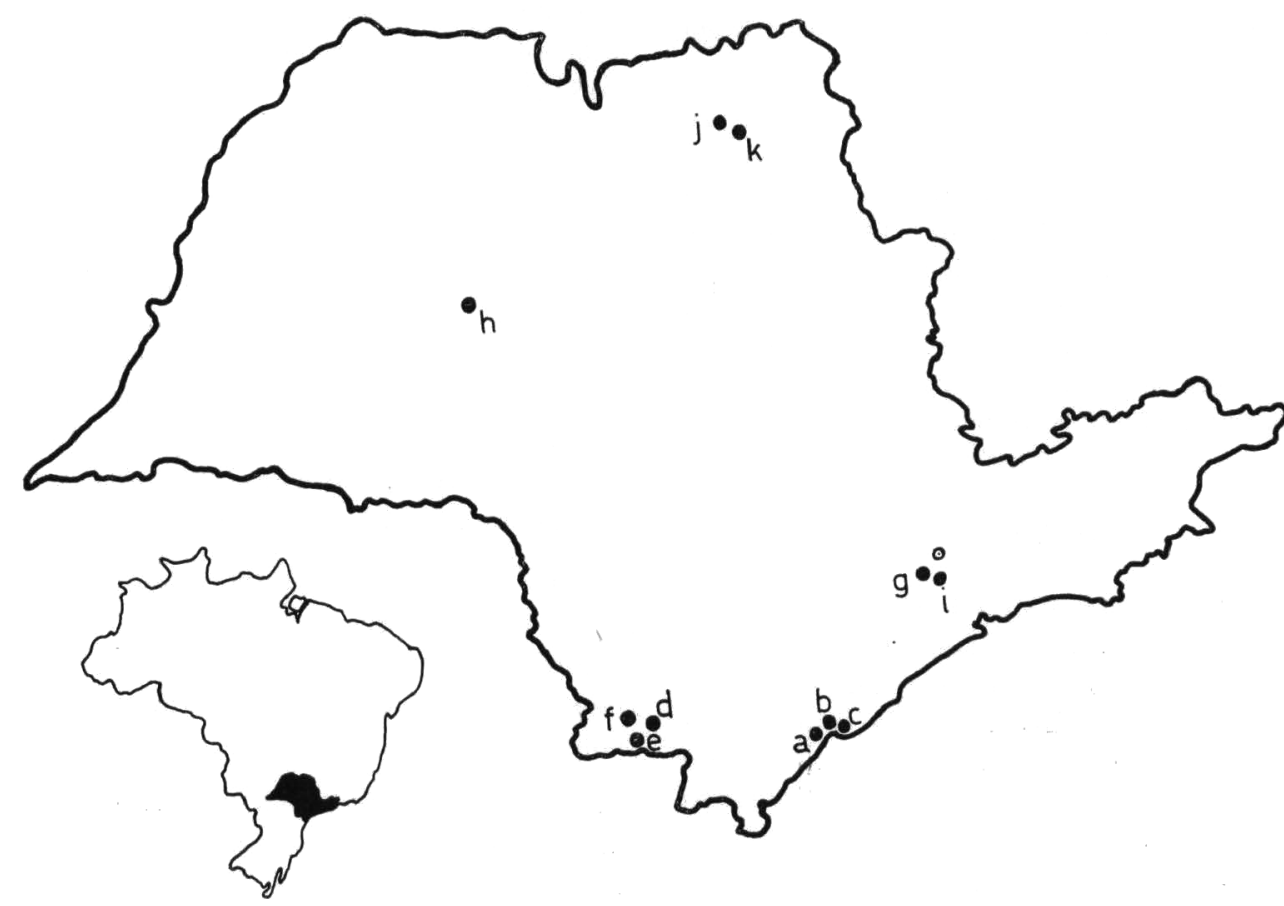

Fig. - Localização das comunidades estudadas, Estado de São Paulo, Brasil - 1969-1973.

$$
\begin{aligned}
& \text { a - Iguape } \\
& \text { b - Icapara } \\
& \text { c - Pontal do Ribeira } \\
& \text { d - Apiaí } \\
& \text { e - Ribeira } \\
& \text { f - Barra do Chapéu }
\end{aligned}
$$

\section{MATERIAL E METODOS}

\section{Amostragem}

Foi realizado um cadastramento em cada uma das comunidades estudadas para estabelecer o numero de familias residentes. Em Icapara e Pontal do Ribeira o inquérito de consumo alimentar foi realizado na tota-

$$
\begin{aligned}
& \text { g - Embu-Guaçu } \\
& \text { h - Getulina } \\
& \text { i - Cipó } \\
& \text { j- Morro Agudo } \\
& \text { k - Salles Oliveira }
\end{aligned}
$$

lidade da população. Nas comunidades restantes, como as populaçōes eram homogêneas, extraiu-se uma amostra casual simples, não-estratificada. Em Morro Agudo e Salles Oliveira os inquéritos clínico e bioquímico foram realizados na mesma amostra dos inquéritos de consumo de alimentos, e nas outras comunidades em sub-amostras do inquérito alimentar. 
RONCADA, M.J. et al. Hiporitaminose A em comunidades do Estado de São Paulo, Brasil. Rev. Saúde públ., S. Paulo, 15:338-49, 1981.

\section{Inquérito de Consumo Alimentar}

O consumo de alimentos foi obtido em duas visitas a cada familia, no mesmo dia. A primeira visita era feita à hora do almoço e todos os alimentos preparados eram pesados; a segunda, era à hora do jantar, tendo sido usado, então, o método recordatório, ıma vez que ambas as refeições tinham praticamente a mesma composição (um traço cultural).

Para o cálculo de calorias e quantidade de nutrientes foi usada a tabela de composição química de alimentos, compilada pelo Departamento de Nutriçãos. Os parâmetros para a adequação da dieta foram as recomendações do National Research Council (NRC) '. A adequação média "per capita" ca dieta foi calculada para cada familia, considerando-se a composição familiar e a presença de cada membro em cada refeição.

\section{Inquérito Bioquimico}

Colheu-se sangue de todos os individuos com idade de 2 anos e mais por venipunção, que foi acondicionado en tubos de cor àmbar contendo EDTA, sendo então tranısportado para o laboratório de campo sob refrigeração. Lá, o sangue era centrifugado imediatamente, o plasma era separado e conservado em congelador a $-18^{\circ} \mathrm{C}$.

No inquérito de 1969 as determinações de vitamina $A$ e caroteno foram realizadas no laboratório de campo. Nos outros inquéritos, elas foram realizadas nos laboratórios do Departamento de Nutrição da FSP/USP, no máximo 15 dias após a coleta. As anostras eram transportadas congeladas. As ttapas de centrifugação, separação e determinação bioquimica foram sempre realizadas em penumbra, para minimizar o efeito da luz.

O método bioquímico utilizado foi o de Carr-Price, realizando controle de qualidade das análises através de amostras-controle, constituidas de plasma homogêneo. Os resultados foram classificados de acordo com os critérios do ICNND ${ }^{5}$.

\section{Inquérito Clinico}

Os sinais clinicos atribuiveis à deficiência de vitamina $A$ que foram pesquisados são: xerose cutânea, hiperceratose folicular, mancha de Bitot, xerose conjuntival, xerose corneal, ceratomalácia, ulceração corneal, perfuração e cicatriz corneal. Os resultados dos exames oculares foram codificados de acordo com o critério recomendado pela OMS/AID 3

\section{RESULTADOS}

\section{Inquérito de Consumo Alimentar}

A Tabela 1 mostra o valor calórico da dieta $e$ as quantidades de proteina total, proteina animal, gordura, vitamina A total e vitamina A pré-formada nos alimentos consumidos nas várias comunidades.

Houve pequena variação nos valores calóricos médios, exceto em Ribeira, o qual apresentou um valor médio de cerca de 750 calorias menor do que o mais alto encontrado (Salles Oliveira).

Com relação à proteína, verificou-se que sua contribuição para o valor calórico total foi uniforme (entre 11 e $13 \%$ ).

Houve grande variação no consumo de gorduras $(23 \mathrm{~g}$ a $80 \mathrm{~g})$, o mesmo acontecendo com a vitamina A $(133 \mu \mathrm{g}$ a $803 \mu \mathrm{g})$.

Distribuindo os resultados em niveis de adequaçāo de calorias e proteinas (Tabela 2), nota-se que uma grande proporção de familias apresentou um nível de adequação abaixo de $80 \%$. Quanto à vitamina $\mathrm{A}$, mais de $80 \%$ das familias apresentaram níveis de adequação abaixo de $60 \%$.

$\mathrm{Na}$ maioria das comunidades o consumo médio diário "per capita" de vegetais e frutas foi insatisfatório, enquanto que em lcapara, Pontal do Ribeira e Ribeira foi insignificante (Tabela 3). 
RONCADA, M.J, et al. Hipovitaminose A em comlinidades do Estado de são Paulo, Brasil. Rev. Saúte públ., S. Paulo. 15:338-49, 1981.

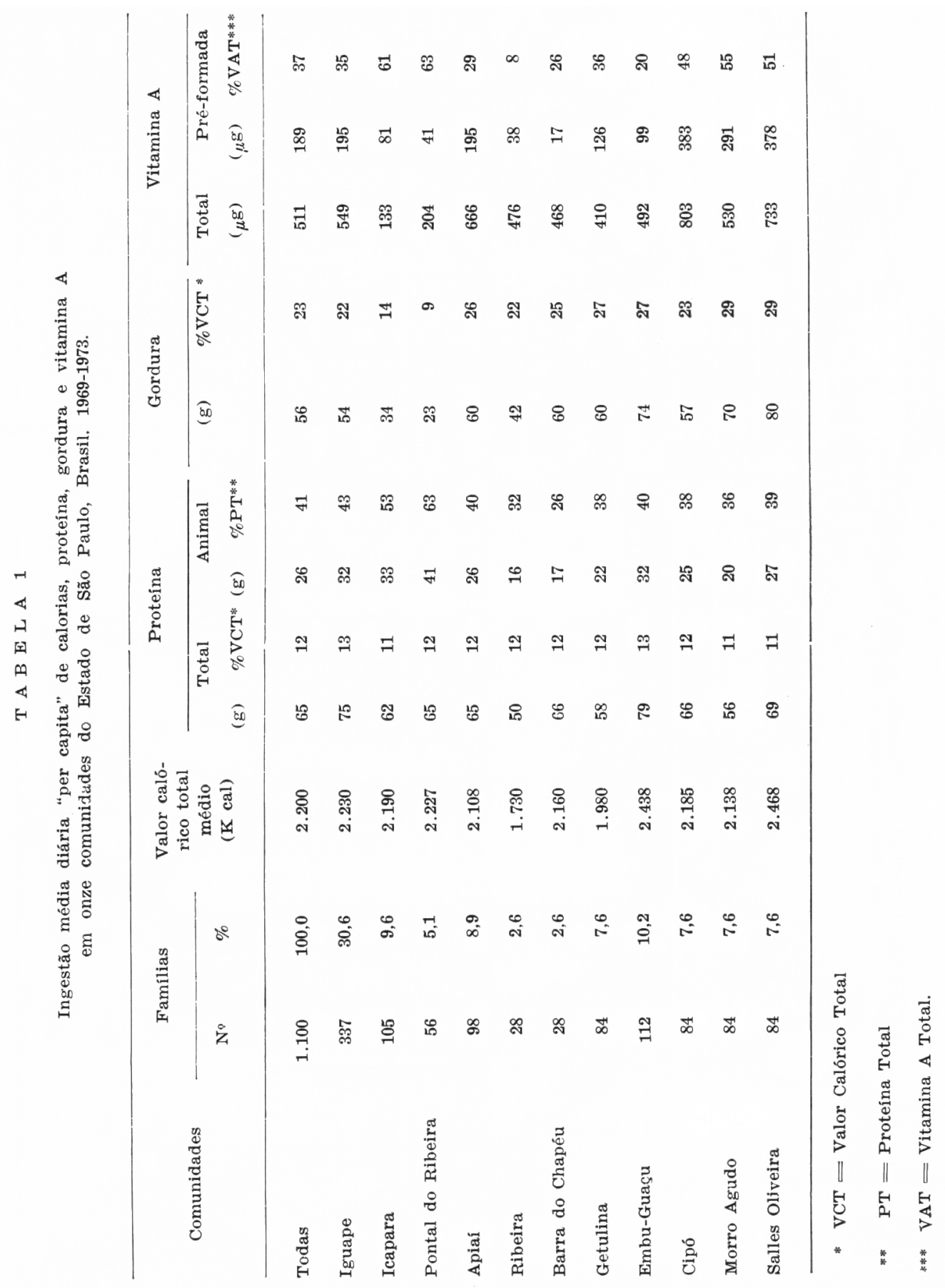




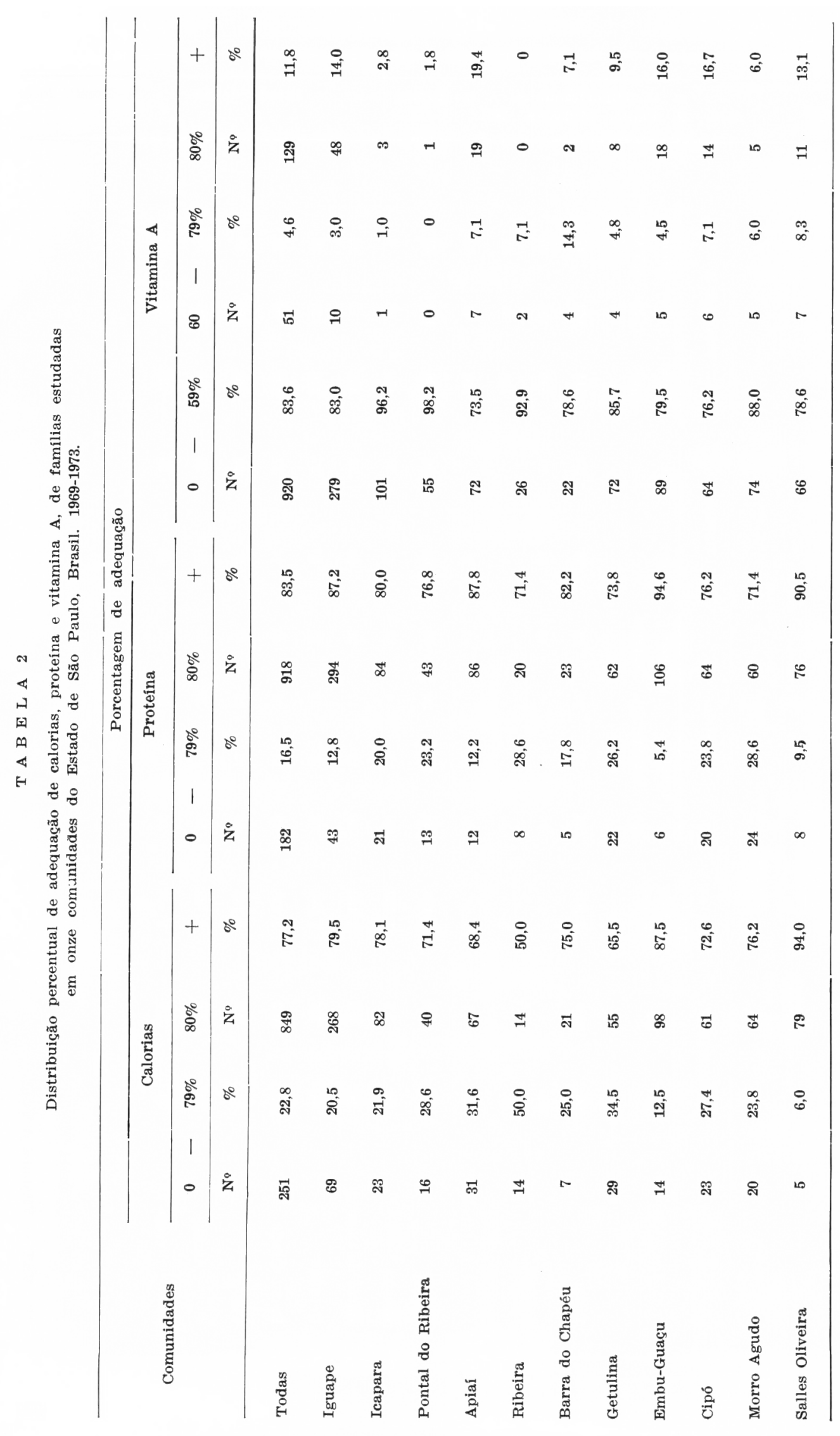


RONCADA, M.J. et al. Hipovitaminose A em comunidades do Estado de São Paulo, Brasil. Rev. Saúde públ., S. Patlo, 15:338-49, 1981.

T A B E L A 3

Consumo médio diário "per capita" de vegetals e frutas, de famílias estudadas em onze comunidades do Estado de São Paulo, Brasil. 1969-1973.

\begin{tabular}{lcc}
\hline \multicolumn{1}{c}{ Comunidades } & $\begin{array}{c}\text { Vegetais } \\
(\mathbf{g})\end{array}$ & $\begin{array}{c}\text { Frutas } \\
(\mathrm{g})\end{array}$ \\
\hline Iguape & 60 & 74 \\
Icapara & 27 & 20 \\
Pontal do Ribeira & 10 & 16 \\
Apiaí & 66 & 96 \\
Ribeira & 36 & 51 \\
Barra do Chapéu & 45 & 49 \\
Getulina & 59 & 85 \\
Embu-Guaçu & 67 & 84 \\
Cipó & 75 & 95 \\
Morro Agudo & 36 & 91 \\
Salles Oliveira & 83 & 139 \\
\hline
\end{tabular}

\section{Inquérito Bioquimico}

As determinações de vitamina A foram realizadas em cerca de $75 \%$ das pessoas examinadas clinicamente. De acordo com a classificação do ICNND s, não se encontrou, em duas das comunidades estudadas, um único indivíduo com valores plasmáticos de vitamina A abaixo de $20 \mu \mathrm{g} / 100 \mathrm{ml}$ (Tabela 4). Com relação ao caroteno (Tabela 5), em duas comunidades não houve resultados classificados como "deficiente" (ICNND); por outro lado, em quatro delas, não foram encontrados resultados considerados "alto".

As mais altas proporções de indivíduos com resultados de vitamina $\mathrm{A}$ plasmática abaixo do normal foram encontradas entre os pré-escolares (Tabela 6, sub-total). 0 mesmo foi observado quanto ao caroteno (Tabela 7).

\section{Inquérito Clinico}

De 1.660 individuos examinados, trezentos e quarenta $(20,5 \%)$ tinham menos de 7 anos de idade.

Considerando as lesões oculares (Tabela 9), independentemente das cutâneas, as proporções variaram entre $15 \%$ e $16 \%$ para xerose conjuntival (X1A), entre $0,0 \%$ e $1,5 \%$ para xerose conjuntival mais mancha de Bitot (X1B) e entre $0,0 \%$ e $1,3 \%$ para xerose conjuntival mais xerose corneal (X2). Os grupos etários com as mais altas proporçōes de lesões oculares foram aqueles de 7 a 10 anos e de 11 a 14 anos de idade. 
TA B E L A 4

Res::ltados das determinaçōes de vitamina A plasmática, segundo classificação do ICNND 5 , em onze comunidades do Estado de São Paulo, Brasil. 1969-1973.

\begin{tabular}{|c|c|c|c|c|c|c|c|c|}
\hline \multirow{3}{*}{ comunidades } & \multicolumn{8}{|c|}{ Niveis de Vitamina A plasmática } \\
\hline & \multicolumn{2}{|c|}{ Deficlente } & \multicolumn{2}{|c|}{ Baixo } & \multicolumn{2}{|c|}{ Aceitável } & \multicolumn{2}{|c|}{ Alto } \\
\hline & No & $\%$ & No & $\%$ & No & $\%$ & Ne & $\%$ \\
\hline Todas & 66 & $\mathbf{5 , 4}$ & 157 & 12,9 & 704 & 57,7 & 294 & 24,1 \\
\hline Iguape & 13 & 6,4 & 8 & 4,0 & 52 & 25,7 & 129 & 63,9 \\
\hline Icapara & 9 & 10,5 & 16 & 18,6 & 50 & 58,1 & 11 & 12,8 \\
\hline Pontal do Ribeira & 6 & 10,9 & 9 & 16,4 & 25 & 45,5 & 15 & 27,3 \\
\hline Apiai & - & - & - & - & 17 & 28,3 & 43 & 71,7 \\
\hline Ribeira & 2 & 3,4 & 1 & 1,7 & 47 & 79,7 & 9 & 15,3 \\
\hline Barra do Chapéu & - & - & - & - & 17 & 43,6 & 22 & 56,4 \\
\hline Getulina & 5 & 3,2 & 12 & 7,6 & 111 & 70,2 & 30 & 19,0 \\
\hline Embu-Guaçu & 10 & 12,6 & 18 & 22,8 & 36 & 45,6 & 15 & 19,0 \\
\hline Cipó & 13 & 11,0 & 34 & 28,8 & 68 & 57,6 & 3 & 2,6 \\
\hline Morro Agudo & 6 & 2,1 & 46 & 16,2 & 221 & $\mathbf{7 7 , 8}$ & 11 & 3,9 \\
\hline Salles Oliveira & 2 & 2,5 & 13 & 16,0 & 60 & $\mathbf{7 4 , 1}$ & 6 & 7,4 \\
\hline
\end{tabular}

T A B E L A 5

Resultados das determinações de caroteno plasmático, segundo classificação do ICNND 5, em onze comunidades do Estado de São Paulo, Brasil. 1969-1973.

\begin{tabular}{|c|c|c|c|c|c|c|c|c|}
\hline \multirow{3}{*}{ Comunidades } & \multicolumn{8}{|c|}{ Niveis de caroteno plasmático } \\
\hline & \multicolumn{2}{|c|}{ Deficlente } & \multicolumn{2}{|c|}{ Baixo } & \multicolumn{2}{|c|}{ Aceitável } & \multicolumn{2}{|c|}{ Alto } \\
\hline & No & $\%$ & No & $\%$ & $\mathrm{~N}^{\circ}$ & $\%$ & Ne & $\%$ \\
\hline Todas & 179 & 14,3 & 258 & 20,7 & 568 & 45,5 & 243 & 19,5 \\
\hline Iguape & 46 & 22,3 & 118 & 57,3 & 42 & 20,4 & - & - \\
\hline Icapara & 70 & 73,7 & 23 & 24,2 & 2 & 2,1 & - & - \\
\hline Pontal do Ribeira & 45 & 78,9 & 11 & 19,3 & 1 & 1,8 & - & - \\
\hline Apiaf & 1 & 1,7 & 12 & 20,0 & 43 & 71,7 & 4 & 6,7 \\
\hline Ribeira & 6 & 10,0 & 24 & 40,0 & 30 & 50,0 & - & - \\
\hline Barra do Chapéu & - & - & 8 & 19,5 & 32 & 78,1 & 1 & 2,4 \\
\hline Getulina & $\mathbf{5}$ & 3,2 & 13 & 8,2 & 96 & 60,8 & 44 & 27,8 \\
\hline Embu-Guaçu & 1 & 1,3 & 8 & 10,1 & 48 & 60,8 & 22 & 27,8 \\
\hline Cipó & 1 & 0,8 & 15 & 12,5 & 64 & 53,3 & 40 & 33,3 \\
\hline Morro Agudo & 4 & 1,4 & 24 & 8,3 & 167 & $\mathbf{5 7 , 6}$ & 95 & 32,7 \\
\hline Salles Oliveira & - & - & 2 & 2,4 & 43 & 52,4 & 37 & 45,1 \\
\hline
\end{tabular}


T A B E L A 6

Resultados das determinações de vitamina A plasmática, segundo idade e classificação do ICNND 5 , em onze comunidades do Estado de São Paulo, Brasil. 1969-1973.

\begin{tabular}{|c|c|c|c|c|c|c|c|c|c|}
\hline \multirow{3}{*}{$\begin{array}{c}\text { Níveis de } \\
\text { Vitamina A } \\
\text { Plasmática }\end{array}$} & \multicolumn{9}{|c|}{ Idade (anos) } \\
\hline & 2 & - & 7 & $-\quad 10$ & 11 & -14 & 15 & + & Todas \\
\hline & $N^{0}$ & $\%$ & $N^{0}$ & $\%$ & No & $\%$ & No & $\%$ & $N^{\circ}$ \\
\hline Deficiente & 17 & 25,8 & 17 & 11,9 & 6 & 3,4 & 26 & 3,1 & 66 \\
\hline Baixo & 15 & 22,7 & 33 & 23,1 & 28 & 15,7 & 81 & 9,7 & 157 \\
\hline S..b.total & 32 & 48,5 & 50 & 35,0 & 34 & 19,1 & 107 & 12,8 & 223 \\
\hline Aceitável & 22 & 33,3 & 82 & 57,3 & 119 & 66,9 & 481 & 57,7 & 704 \\
\hline Alto & 12 & 18,2 & 11 & 7,7 & 25 & 14,0 & 246 & 29,5 & 294 \\
\hline Sub-total & 34 & 51,5 & 93 & 65,0 & 144 & 80,9 & 727 & 87,2 & 998 \\
\hline Total & 66 & $\begin{array}{l}100,0 \\
(5,4) *\end{array}$ & 143 & $\begin{array}{c}100,0 \\
(11,7)^{*}\end{array}$ & 178 & $\begin{array}{c}100,0 \\
(14,6) \text { * }\end{array}$ & 834 & $\begin{array}{c}100,0 \\
(68,3) \text { * }\end{array}$ & 1.221 \\
\hline
\end{tabular}

* Os números colocados entre parenteses representam as porcentagens em relação ao total geral de examinados -1221.

\section{T A B E L A 7}

Resultados das determinações de caroteno plasmático, segundo idade e classificação do ICNND 5 , em onze comunidades do Estado de São Paulo, Brasil. 1969-1973.

\begin{tabular}{|c|c|c|c|c|c|c|c|c|c|}
\hline \multirow{3}{*}{$\begin{array}{l}\text { Níveis de } \\
\text { Caroteno } \\
\text { Plasmático }\end{array}$} & \multicolumn{9}{|c|}{ Idade (anos) } \\
\hline & $2-$ & 6 & $7-$ & 10 & $11-$ & 14 & 15 & + & Todas \\
\hline & No & $\%$ & $\mathrm{~N}^{\circ}$ & $\%$ & $N$ & $\%$ & No & $\%$ & $\mathrm{~N}^{\circ}$ \\
\hline Deficiente & 23 & 32,4 & 22 & 14,6 & 21 & 11,5 & 113 & 13,4 & 179 \\
\hline Baixo & 18 & 25,4 & 26 & 17,2 & 33 & 18,1 & 181 & 21,4 & 258 \\
\hline Sub-total & 41 & 57,7 & 48 & 38,1 & 54 & 29,7 & 294 & 34,8 & 437 \\
\hline Aceitável & 20 & 28,2 & 71 & 47,0 & 90 & 49,5 & 387 & 45,9 & 568 \\
\hline Alto & 10 & 14,1 & 32 & 21,2 & 38 & 20,9 & 163 & 19,3 & 243 \\
\hline Sub-total & 30 & 42,3 & 103 & 68,2 & 128 & 70,3 & 550 & 65,2 & 811 \\
\hline Total & 71 & $\begin{array}{l}100,0 \\
(5,7) *\end{array}$ & 151 & $\begin{array}{c}100,0 \\
(12,1) *\end{array}$ & 182 & $\begin{array}{c}100,0 \\
14,6) *\end{array}$ & 844 & $\begin{array}{c}100,0 \\
(67,6)^{*}\end{array}$ & 1.248 \\
\hline
\end{tabular}


RONCADA, M.J. et al. Hipovitaminose A em cominidades do Estado de São Paulo, Brasil. Rev. Saúde públ., S. Paulo, 15:338-49, 1981.

\section{COMENTARIOS E CONCLUSOES}

Embora muitas famílias nas comunidades estudadas pertencessem a um baixo nivel sócio-econômico, a ingestão calórica foi adequada na maioria delas (Tabela 2).

Os resultados referentes ao consumo de proteinas pode ser considerado satisfatório, pois uma grande proporção de famílias estudadas situou-se nos níveis mais altos (Tabela 2).

A proporção de proteina de origem animal em relação à proteina total foi também satisfatória (Tabela 1), especialmente nas áreas litorâneas, onde $o$ peixe e a farinha de mandioca eram os alimentos básicos.

Quanto à vitamina A, o oposto foi observado. As médias diárias "per capita" foram baixas em todas as comunidades, exceto uma (Cipó). Isso porque, ai uma familia consumiu $1,5 \mathrm{~kg}$ (cerca de $300 \mathrm{~g}$ "per capita") de fígado no dia em que foi visitada. $O$ fato, naturalmente, aumentou o consumo médio diário "per capita" nessa comunidade. Entretanto, o consumo de tão grandes quantidades de fígado não é usual, não somente considerando comunidades, como também esta família em particular. Este acontecimento é uma das falhas onde o consumo médio "per capita" é usado em estudos de comunidade.

Embora o consumo de vitamina A tenha sido baixo, cerca de $30 \%$ foi de vitamina A pré-formada em $\mathbf{7 3} \%$ das comunidades estudadas. Em comunidades onde 0 peixe é o alimento básico, o consumo de vitamina A pré-formada foi até mais alto $(60 \%$ da atividade total de vitamina $A$ ). Provavelmente tal fato se deve ao hábito de consumir pequenos peixes sem remover as visceras 4 .

Em relação à proteina e gordura, os resultados indicam um equilibrio relativamente adequado entre os elementos energéticos que compōem a dieta, excetuando-se Icapara e Pontal do Ribeira, onde o consumo de gordura foi acentuadamente baixo. E pos- sivel que um consumo satisfatório de gordura $(22 \%$ a $29 \%)$ em nove comunidades seja responsável por uma melhor absorção de vitamina $A$.

Considerando os dados bioquímicos das onze comunidades como um todo (Tabela 4), podemos admitir que a deficiência de vitamina A é um problema de Saúde Pública, pois 5,4\% dos indivíduos apresentaram niveis sangüineos de $10 \mu \mathrm{g} / 100 \mathrm{ml}$ ou menos, e $18,3 \%$, de $20 \mu \mathrm{g} / 100 \mathrm{ml}$ ou menos (de acordo com o critério do ICNND).

Entretanto, considerando as comunidades isoladamente, pode-se observar que 4 delas não apresentaram niveis críticos com relação à carência de vitamina $A$.

Distribuindo os resultados de acordo com a idade (Tabela 6 ), torna-se evidente que os pré-escolares apresentaram os coeficientes mais altos de resultados tanto abaixo de $10 \mu \mathrm{g} / 100 \mathrm{ml}$ como abaixo de $20 \mu \mathrm{g} / 100 \mathrm{ml}$. Esses coeficientes foram tanto menores quanto maior a idade.

Os niveis plasmáticos de caroteno são indices úteis para estimar o estado nutricional de uma população com relação à vitamina $A$, pois a maioria dos indivíduos depende, em grande parte, deste precursor; e os níveis plasmáticos de caroteno referem-se à ingestão recente de vegetais ricos neste pigmento 2. Tanto o inquérito bioquímico, como 0 de consumo de alimentos (Tabelas 5 e 3) indicam uma baixa ingestão de verduras e frutas consideradas ricas em caroteno. Nas três comunidades litorâneas nenhum resultado "alto" foi obtido, ao passo que a prevalência de resultados abaixo de $40 \mu \mathrm{g} / 100 \mathrm{ml}$ foi alta. Estes resultados não são surpreendentes, uma vez que essas comunidades são habitadas principalmente por pescadores, cuja alimentação básica é peixe e farinha de mandioca ${ }^{4}$; o consumo de alimentos ricos em caroteno foi muito baixo: vegetais mais frutas correspondiam a $47 \mathrm{~g}$ diárias "per capita" em Icapara, 26g em Pontal do Ribeira e $137 \mathrm{~g}$ em Iguape; 
RONCADA, M.J. et al. Hipovitaminose A em comunidades do Estado de São Paulo, Brasil. Rev. Saude públ., S. Paulo, 15:338-49, 1981.

a maioria das frutas era constituída por bananas, já que essa região é a maior produtora dessa fruta no Estado de São Paulo. Ribeira, que também não apresentou resultados de caroteno plasmático classificados como "alto" era, na ocasião, a maior produtora de mamão no Estado, embora quase a totalidade da produção fosse exportada para outras cidades, especialmente a Capital, o que originou um consumo insignificante 1. Inversamente, Salles Oliveira mostrou o maior consumo "per capita" de vegetais e frutas $(222 \mathrm{~g})$ e nenhum resultado "deficiente".

Apesar disso, os resultados apresentados devem ser considerados com cautela, pois os resultados bioquímicos são individuais $\mathrm{e}$ os referentes ao consumo de alimentos representam médias familiares.

As maiores proporções de resultados "deficiente" (Tabela 7), referentes ao caroteno, ocorreram entre crianças de 2 a 6 anos de idade, imediatamente seguidas por aquelas de 7 a 10 anos de idade. Isso sugere que o consumo de vegetais e frutas é baixo entre as crianças, talvez devido a crenças populares relacionadas a estes alimentos, que limitariam seu uso.

Esses fatos levam-nos a acreditar que o baixo consumo de vegetais e frutas por nossas populações se devem à sua ignorância no que concerne ao valor nutricional desses alimentos, uma vez que eles são muito mais baratos e mais disponíveis do que as fontes da vitamina A pré-formada. A importância da educação nutricional é óbvia.

Os dados apresentados na Tabela 8 mostram que a maioria dos sinais clínicos apresentados pelos individuos são cutâneos, sem lesăo ocular. Entretanto, os coeficientes para as lesões oculares não são baixos. Considerando os grupos etários, não houve diferenças significantes entre os coeficientes de prevalência.
Considerando os sinais clinicos oculares atribuiveis à deficiência de vitamina $A$, vê-se claramente que a xerose conjuntival (X1A) é, incontestavelmente, o sinal clínico mais prevalente e igualmente distribuido por todos os grupos etários. Mancha de Bitot mais xerose conjuntival $(\mathrm{X} 1 \mathrm{~B})$ foram pouco prevalentes, assim como xerose conjuntival mais xerose corneal, X2 (Tabela 9).

Embora os dados bioquímicos mostrem que a hipovitaminose $A$ é um problema de Saúde Pública, os dados clínicos mostram que a xeroftalmia não o é (critério da OMS).

Entretanto, outros aspectos devem ser considerados. Com relação à história natural da deficiência de vitamina $A$, temos que reconhecer que a xeroftalmia representa uma conseqüência final $\mathrm{da}$ deficiência. Quando a xeroftalmia se apresenta como um problema, a deficiência de vitamina A subjacente se apresenta como um problema muitas vezes maior. Inversamente, quando a deficiência de vitamina $\mathrm{A}$ se apresenta como um problema de Saúde Pública, embora a xeroftalmia não se apresente, seu aparecimento será apenas uma questão de tempo e, se medidas não forem tomadas, a populaçăo ficará exposta a um risco cada vez maior no que diz respeito à cegueira.

Considerando-se os nossos dados, concluimos que, nessas comunidades:

- foi baixo o consumo tanto de fontes de vitamina A pré-formada, como da pró-vitamina;

- foi alta a prevalência de niveis plasmáticos de vitamina A classificados como "deficiente" e "baixo";

- os sinais clínicos cutâneos da deficiência de vitamina $\mathrm{A}$ apresentaram alta prevalência, $\mathrm{o}$ inverso acontecendo com os oculares. 
RONCADA, M.J. et al. Hipovitaminose A em comunidades do Estado de Săo Paulo, Brasil. Rev. Saúde puibl., S. Pa:lo, 15:338-49, 1981.

T A B E L A 8

Frequência de indivíduos apresentando sinais clínicos atribuiveis à hipovitaminose A em relação a pessoas examinadas, segundo ldade, em onze comunidades do Estado de São Paulo, Brasil. 1969-1973.

\begin{tabular}{|c|c|c|c|c|c|c|c|c|c|}
\hline & \multirow{3}{*}{$\begin{array}{l}\text { Idade } \\
\text { (anos) }\end{array}$} & \multicolumn{8}{|c|}{ Sinais clinicos } \\
\hline & & \multicolumn{2}{|c|}{$\begin{array}{l}\text { Somente } \\
\text { cutâneos }\end{array}$} & \multicolumn{2}{|c|}{$\begin{array}{l}\text { Oculares mais } \\
\text { cutâneos }\end{array}$} & \multicolumn{2}{|c|}{$\begin{array}{l}\text { Somente } \\
\text { oculares }\end{array}$} & \multicolumn{2}{|c|}{$\begin{array}{c}\text { oculares } \\
\text { Totais }\end{array}$} \\
\hline & & No & $\%$ & No & $\%$ & $\mathrm{No}$ & $\%$ & No & $\%$ \\
\hline & das & 849 & 51,1 & 157 & 9.5 & 114 & 6.9 & 271 & 16,3 \\
\hline 0 & -6 & 122 & 35.9 & 26 & 7,6 & 29 & 8,5 & 55 & 16,2 \\
\hline 7 & -10 & 130 & 57,3 & 22 & 9,7 & 17 & 7,5 & 39 & 17,2 \\
\hline 11 & -14 & 104 & 51,7 & 20 & 10,0 & 15 & 7,5 & 35 & 17,4 \\
\hline 15 & + & 493 & 55,3 & 89 & 10,0 & 58 & 5,9 & 142 & 15,9 \\
\hline
\end{tabular}

T A B E L A 9

Frequência dos diferentes sinais clinicos oculares atribuiveis à hipovitaminose A. nos 271 individuos que os apresentam, com e sem sinais cutaneos, segundo idade, em onze comunidades do Estado de Săo Paulo, Brasil. 1969-1973.

\begin{tabular}{|c|c|c|c|c|c|c|c|}
\hline & \multirow{3}{*}{$\begin{array}{l}\text { Idade } \\
\text { (anos) }\end{array}$} & \multicolumn{6}{|c|}{ Sinais clínicos oculares } \\
\hline & & \multicolumn{2}{|c|}{$\begin{array}{c}\text { Xerose } \\
\text { conjuntival } \\
\text { (X1A) }\end{array}$} & \multicolumn{2}{|c|}{$\begin{array}{c}\text { Mancha de Bitot com } \\
\text { Xerose conjuntival } \\
\text { (X1B) }\end{array}$} & \multicolumn{2}{|c|}{$\begin{array}{c}\text { Xerose corneal com } \\
\text { Xerose conjuntival } \\
(\mathrm{X} 2)\end{array}$} \\
\hline & & Ne & $\%$ & No & $\%$ & No & $\%$ \\
\hline Toc & das & 256 & 15,5 & 9 & 0,5 & 6 & 0,4 \\
\hline c & -6 & 51 & 15,0 & 3 & 0.9 & 1 & 0,3 \\
\hline 7 & -10 & 36 & 15,9 & $\mathbf{0}$ & - & 3 & 1,3 \\
\hline 11 & -14 & 32 & 15,9 & 3 & 1,5 & 0 & - \\
\hline 15 & + & 137 & 15,4 & 3 & 0.3 & 2 & 0,2 \\
\hline
\end{tabular}


RONCADA, M.J. et al. Hipovitaminose A em comunidades do Estado de São Paulo, Brasil. Rev. Saúde públ., S. Pat:lo, 15:338-49, 1981.

RONCADA, M.J. et al. [Vitamin A deficiency in communities of the State of $\mathbf{S}$. Paulo, Brazil]. Rev. Saúde públ., S. Paulo, 15:338-49, 1981.

ABSTRACT: Eleven communities of the State of $\mathbf{S}$. Paulo, Brazil, were surveyed for vitamin A deficiency, through food consumption survey, clinical survey and biochemical survey. The food consumption survey showed very low consumption of vitamin A rich foods, both animal and vegetable. The biochemical survey showed high prevalence of low and deficient plasma levels of vitamin A (ICNND classification). The clinical survey showed low prevalence rates for ocular lesions, specially the more severe ones. Although neither blindness nor severe ocular lesions are a Public Health problem the majority of populations are at risk of such lesions becoming a problem in the future.

UNITERMS: Vitamin A deficiency. Nutrition surveys. State of $\mathbf{S}$. Paulo, Brazil. Xerophthalmia.

\section{REFERENCIAS BIBLIOGRAFICAS}

1. BON, A.M.X. \& MIGUEL, M. O consumo de vitamina A em Ribeira, São Paulo (Brasil). Rev. Saúde públ., S. Paulo, $8: 87-92,1974$.

2. PEARSON, W.N. Biochemical appraisal of nutritional status in man. Amer. J. clin. Nutr., 11:462-76, 1962.

3. REUNION CONJUNTA OMS/AID SOBRE CARENCIA DE VITAMINA A $Y$ XEROFTALMIA, Yakarta, 1974. Informe. Ginebra, Organización Mundial de la Salud, 1976. (OMS - Ser. Inf. teen., 590).

4. RONCADA, M.J. Hipovitaminose A. Niveis séricos de vitamina $A$ e caroteno em populações litorâneas do Estado de São Paulo, Brasil. Rev. Saúde públ., $\mathbf{S}$. Paulo, 6:3-18, 1972.

5. UNITED STATES. Interdepartmental Committee on Nutrition for National Defense. Manual for nutrition surveys. 2nd ed.
Washington, D.C., Government Printing Office, 1963.

6. UNITED STATES. Interdepartmental Committee on Nutrition for National Development. Northeast Brazil, nutrition survey: March-May 1963: a report. Washington, D.C., 1965.

7. UNITED STATES. National Academy of Sciences. Recommended dietary allowances: report. 7 th ed. Washington, D.C., 1968.

8. UNIVERSIDADE DE SÃO PAULO. Faculdade de Saúde Pública. Departamento de Nutrição. Tabela de composição quimica dos alimentos: compilação de várias tabelas nacionais $e$ estrangeiras. São Paulo, 1971. [mimeografado].

Recebido para publicasão em 08/08/1980 Aprovado para publicą̧ão em 15/05/1981 